\title{
Historia de la educación en Chihuahua: el Seminario de Microhistoria
}

\author{
History of education in Chihuahua: The Microhistory Seminar
}

\author{
Francisco Alberto Pérez Piñón • Guillermo Hernández Orozco • Stefany Liddiard Cárdenas
}

Francisco Alberto Pérez Piñón. Universidad Autónoma de Chihuahua, México. Es doctor en Ciencias Pedagógicas (Cuba). Entre sus publicaciones recientes están "La hacienda de Humboldt en Chihuahua, lugar de refugio para los bóers precedentes de Sudáfrica a principios del siglo XX" (2019) y "Cuba en movimiento: derrumbe del socialismo, una mirada de primer orden" (2018). Desarrolla la línea de investigación de Historia e Historiografía de la Educación. Cuenta con reconocimientos Prodep y del Sistema Nacional de Investigadores, nivel 1. Es miembro de la Sociedad Mexicana de Historia de la Educación, del Consejo Mexicano de Investigación Educativa y de la Red de Investigadores Educativos Chihuahua. Correo electrónico: aperezp@uach.mx. ORCID: https:// orcid.org/0000-0003-4316-6484.

Guillermo Hernández Orozco. Universidad Autónoma de Chihuahua, México. Es doctor en Ciencias de la Educación por el Instituto Superior Pedagógico Enrique José E. Varona, Cuba. Entre sus publicaciones recientes están "La formación de investigadores en Chihuahua: una mirada histórica" (2019) y "Universidad Autónoma de Chihuahua, el contexto durante su creación en 1954" (2019). Cuenta con reconocimientos al Perfil Prodep y del Sistema Nacional de Investigadores, nivel 1. Sus temas de interés son la historia e historiografía de la educación. Correo electrónico: ghernand@uach.mx. ORCID: https://orcid.org/0000-0001-7287-8240.
Resumen

Como parte del proyecto de Historia de la Educación que se desarrolla en el doctorado en Educación, Artes y Humanidades de la Facultad de Filosofía y Letras de la Universidad Autónoma de Chihuahua, se contempla el Seminario de Microhistoria, impartido a estudiantes del cuarto semestre, con el fin de que blinden teórica y metodológicamente sus trabajos de investigación, que realizan al cruce del currículo. En este artículo se realiza la narrativa y las experiencias obtenidas en el desarrollo de las actividades instrumentadas en el seminario; incluyendo la selección de los artículos científicos a revisar -y que a manera de texto-pretexto sirvieron para la generación de la discusión-, así como la forma metodológica para su abordaje, con un liderazgo compartido con los participantes profesores y estudiantes. La regla es el respeto a las ideas de los demás, con el principio de "quien más habla, más se equivoca y quien no habla, nunca se equivoca", para motivar la participación y perder el miedo a los equívocos. El sustento epistemológico se dejó claro, fue el de generar ideas provenientes de las lecturas y adicionar las comprensiones e interpretaciones que cada uno de los participantes integrara, de conformidad con la utilidad del conocimiento construido. Lo que en el escrito se menciona es lo concerniente a cómo se trabajaron las diferencias entre la microhistoria de las vertientes mexicana e italiana, ante la imposibilidad de describir todo el contenido abordado en el seminario.

Palabras clave: Historia de la educación, microhistoria a la italiana, microhistoria a la mexicana, seminario de microhistoria.

Abstract

As part of the project of History of Education developed in the Doctorate in Education, Arts and Humanities of the Faculty of Philosophy and Letters of the Universidad Autónoma de Chihuahua, students of fourth semester take the course named Microhistory Seminar. Its purpose is to theoretically 
Stefany Liddiard Cárdenas. Universidad Pedagógica Nacional del Estado de Chihuahua, México. Es doctora en Educación, Artes y Humanidades por la Universidad Autónoma de Chihuahua. Sus investigaciones han abordado temas relacionados con la historia e historiografía de la educación. Actualmente es profesora-investigadora de la Universidad Pedagógica Nacional del Estado de Chihuahua. Es miembro del Sistema Nacional de Investigadores del Consejo Nacional de Ciencia y Tecnología, nivel Candidato; miembro asociado candidato del Consejo Mexicano de Investigación Educativa; socia activa de Red de Investigadores Educativos Chihuahua y de la Sociedad Mexicana de la Historia de la Educación. Correo electrónico: sliddiard@upnech.edu.mx. ORCID: https:// orcid.org/0000-0002-3234-4372. and methodologically strengthen their research work, which they develop at the crossroads of the curricula. This article presents the narrative and the experiences obtained in the development of the activities implemented in the seminar. It includes the selection of the scientific articles reviewed and as a text-pretext served to generate the discussion and other papers not considered in the program. The methodological approach was also considered, based on leadership shared with the participants, teachers and students, and always making sure to respect the ideas of others, with the principle of "whoever speaks more, makes more mistakes and whoever does not speak, never makes one". The objective for that was to motivate participation and lose the fear of making mistakes. The epistemological support was made clear, it was to generate ideas coming from the readings, and to add the comprehensions and interpretations integrated by each one of the participants, in accordance with the usefulness of the knowledge they built. Also mentioned in the paper are the concerns about how the differences between the microhistory of the Mexican and the Italian side were approached, given the impossibility of describing the whole seminar. Keywords: History of education, Italian microhistory, Mexican microhistory, microhistory seminar.

\section{INTRODUCCIÓN}

El doctorado en Educación, Artes y Humanidades es un programa acreditado por el Consejo Nacional de Ciencia y Tecnología (CONACyT), abocado a la investigación en las tres áreas, como lo indica su nombre. Como parte del área de educación está la línea de historia de la educación, por lo que se forma a los doctorandos con elementos teóricos y metodológicos relacionados con este tipo de investigación, para lo cual existe un espacio curricular denominado Seminario de Microhistoria, que es el objeto de trabajo del presente escrito. Se describen las actividades realizadas en este espacio curricular y en concreto de una de las temáticas, ante la imposibilidad de hacerlo sobre la totalidad, debido a la abundancia de temas programados y los que iban surgiendo como resultado de la discusión. El objetivo fue dar respuesta al problema que representa para los estudiantes establecer los enfoques teóricos y metodológicos de su objeto de la investigación, y a través del seminario se busca llenar ese vacío en su formación investigativa.

$\mathrm{Al}$ inicio de las actividades se establecieron las reglas para abordar los textos previamente seleccionados, en su mayoría artículos arbitrados o científicos atingentes a la microhistoria. Se determinó la participación rotativa y la forma de coordinar las actividades.

Uno de los temas a tratar fue la diferencia que existe entre lo que se ha venido considerando la microhistoria a la mexicana y la microhistoria a la italiana, teniendo forzosamente que abordar las obras del historiador Luis González y González para la primera postura y a Carlo Ginzburg para la segunda. Se rescatan los elementos 
que identifican a cada una de las vertientes; sin embargo, la finalidad de realizar este ejercicio tiene que ver con la forma como los doctorandos utilizan los elementos discutidos y su posterior incorporación en los trabajos de investigación que desarollan. Adicionalmente se aborda en el escrito el paradigma denominado Indiciario, del que se parte para realizar estudios de microhistoria. El seminario contó con una duración de 48 horas al semestre.

\section{CONTENIDO}

Para el desarrollo del Seminario de Microhistoria se plantearon los elementos teóricos y metodológicos que sustentan la vertiente de lo que se conoce como Microbistoria -a partir de la década de 1970- y que se refiere a una de las formas que emergieron a la caída de los paradigmas macrohistóricos. Estos explicaban el desarrollo social como una totalidad y las partes se consideraban una manifestación concreta de esa totalidad en la unidad, por lo que llegó a negarse -O al menos permanecieron invisibles- las historias locales o regionales y prevalecen las historias generales y universales. Bajo esta tendencia se perdió la fuerza constructiva del artífice de los cambios sociales: el sujeto, habitante de los espacios micro, del pueblo o de las comunidades, quien es ahora el punto nodal de las nuevas narrativas de la historia micro.

En relación a la metodología, se rescatan y se describen los métodos que enriquecen los procedimientos de esta nueva vertiente que privilegia esa mirada desde abajo, desde lo más cercano a nosotros. Tanto lo teórico como lo metodológico de este paradigma, como así se le considera, forma parte de la narrativa y de las actividades desarrolladas en el Seminario de Microhistoria.

$\mathrm{Al}$ inicio de los trabajos se dialogó con los participantes para establecer los objetivos y las metas durante el inicio, desarrollo y al término del seminario, así como los compromisos y las tareas a realizar por el colectivo que estaría participando. Como parte de la dinámica del trabajo, se socializó la idea de que las actividades a realizar eran de manera compartida; cada uno de los participantes debía leer los contenidos seleccionados para las sesiones de trabajo, realizar la interpretación y vincular los contenidos en su proceso investigativo. Esta última parte representaba la fase de utilidad y valor agregado. No se soslayó que el término "seminario" proviene de la semilla o el semen de ideas que se ponen en común y cada uno de los participantes las internaliza y acomoda, dependiendo de sus fines, lo que encarna la forma epistemológica de construcción del conocimiento (constructivismo), como teóricamente lo mencionan las teorías cognoscitivistas de este corte.

Los artículos fueron seleccionados de una amplia gama de opciones de acceso abierto en la red, con la condición de que provinieran de revistas indizadas y que propusieran reflexiones interesantes. Se buscó que no fueran muy extensos, pero sí muy atingentes a la microhistoria, en lo concerniente a los elementos teóricos, metodológicos y casos ejemplares. 
Se realizaron explicitaciones introductorias relacionadas con las fuentes, para que se tomaran en cuenta los deseos y aspiraciones de quienes las escriben, o -como diría Paul Ricoeur (citado en Lafuente, 1998)- el sentido, lo humano que está presente en los escritos, considerando que no son solo papeles para leer sino que es necesario hacer esos rodeos intelectuales para descubrir la esencia, lo oculto, lo que no es explícito, pero hay que intuirlo.

En este punto se hizo explícito que en el caso de la investigación histórica es muy factible que el análisis del pasado se realice a partir de las fuentes, pues es la única forma de reconstruir los acontecimientos, y a la vez representan la memoria que ha quedado impresa en documentos, los cuales luego clasificamos como fuentes primarias o secundarias. Pero no solo los papeles contienen las aspiraciones de quienes nos antecedieron y tampoco son una representación fiel de lo ocurrido. Es necesario contextualizar y concatenar unas fuentes con otras, a manera de verificación, para no dejarnos llevar por el canto y el encanto del encuentro de intersubjetividades del pasado y el presente. No se soslayó que trabajar con el pasado temporalmente distante puede dejar al investigador atrapado en los deseos que como humanos queremos: una sociedad igualitaria, la economía como reparto de lo producido de manera equitativa y una política más democrática.

Se discutió y concluyó que las evidencias escritas y almacenadas en los archivos no son solo bultos de papeles, representan la memoria de personas de carne y hueso que tuvieron idealizaciones para direccionar a la sociedad, en síntesis, las fuentes son el concentrado de los desiderátums humanos. También -y debido a las intervenciones de los estudiantes del seminario- la lluvia de ideas se fue perfilando a la necesidad de considerar que la historia no es solo esa disciplina que se ocupa del pasado, sino que también es su ocupación el tiempo presente. Para ello, de nueva cuenta, se dio la discusión con el entramado de que ya es tiempo de considerar a la historia como lo que es: una ciencia social, esto quiere decir que está referida al rescate de las acciones humanas del pasado y del presente, y que no tiene caso rescatar acontecimientos tan lejanos en lo temporal que carezcan de relación con el presente, pues solo servirían como material para el coleccionista de antigüedades que se dedica a almacenar "cositas raras".

La discusión fue apasionante y se retomó como hilo conductor para el análisis e interpretación de las lecturas seleccionadas para el seminario: la historia como disciplina que se ocupa del tiempo pasado y del tiempo presente; adentrándonos en ese afluente de frescura que ha venido a renovar la disciplina, con los argumentos posmodernistas de autores como Hayden White y Keith Jenkins. Ellos llegaron al extremo de negar los acontecimientos en el tiempo, con frases como "el pasado ya no existe y es imposible su reproducción para el análisis", así que lo único que tenemos del pasado son capas y capas de interpretaciones (Jenkins, 2006), o llegaron a mencionar que la historia se reduce solo a la escritura, generando así lo que conocemos como el giro lingüístico de la historia (White, 1992). 
Una de las lecturas analizadas fue la que se refiere a la mesa redonda coordinada por López (2005) "Microhistoria mexicana, microhistoria italiana e historia regional", de la que previamente se aclaró que era el pretexto para iniciar la discusión y la producción de ideas, que se centraron en la diferenciación de lo que es la línea de la microhistoria a la mexicana y a la italiana. A través del texto-pretexto se tuvo la oportunidad de profundizar en las obras de don Luis González y González, considerado el padre de la microhistoria a la mexicana, de las cuales rescatamos algunas ideas eje de la discusión:

...estudiar aquellos aspectos de la vida que están más allá de las estatuas de bronce y el interés por los grandes negocios o por los grandes hombres. Es decir: utilicé el término para referirme a la vida cotidiana de un ser en su propio medio, para hablar del hombre común y corriente (de estatura normal, no de los "gigantes" como hace la historia normalmente), de los modos de proceder que son los más íntimos, pero también los más propios del ser humano en general [citado en López, 2005, p. 198].

La cita anterior hace alusión a la escritura de la obra de San José de Gracia, para justificar por qué utilizó el término de "microhistoria". El mismo autor señala que fue para nombrar a esa historia que siempre queda desconocida, olvidada y opacada por las grandes historias que-como lo mencionamos al inicio de la narrativa-borran las acciones de los hombres y mujeres de carne y hueso, haciéndola parecer como una historia desconocida para el mismo ser que forma parte de ella.

La discusión versó en relación a que la disciplina histórica debe rescatar las acciones cotidianas, esos intercambios de comunicación que día a día se establecen entre las personas. Debe recuperar eso que también es objeto de estudio de la historia (orientarse al estudio de las mentalidades), la cultura e idearios de los seres humanos que no deben dejarse de lado. Los comentarios también se perfilaron al tema de las fronteras de las ciencias, a partir de la pregunta explícita de los miembros del seminario, argumentando si no se estaba invadiendo la frontera de la sociología. La discusión fue abundante, pero en este espacio de escritura solo se expresa que fue necesario hacer el llamado a la interdisciplinariedad, a la reflexión de que cuando se abordan los objetos de estudio de manera unidisciplinar se quedan muy reducidos los resultados, de ahí la necesidad de apoyarse en otras disciplinas que permitan profundizar en lo investigado. Estuvo presente - por supuesto- la teoría de la complejidad, como ese entramado social al cual hay que analizar e interpretar. ¿Qué sería de la historia sin el apoyo de otras disciplinas? Quedaría reducida a lo que durante tantos años se desplazó, a narraciones de los hechos del pasado, que hoy no se refiere de manera exclusiva al estudio del pasado, ni son hechos, como nos lo hacía ver Durkheim; se trata de acontecimientos, deseos, aspiraciones y voliciones de las personas en su tiempo. Por ello, cuando se tienen los documentos y se concatenan, se deben hacer esos rodeos intelectuales para llegar a la esencia y no quedarse solo con la apariencia.

Continuando con otra idea de Luis González, nos menciona que: 
Yo no utilicé el término microhistoria para hablar de la historia local (aunque, después de todo, todas las historias son locales en cuanto a que suceden en algún lugar), sino porque estimé que buscaba ciertos fondos del ser humano que no aparecen en la historia nacional, mucho menos en la filosofía de la historia [citado en López, 2005, p. 198].

Con esta cita nos remonta a la necesidad de ubicar el nivel micro en el universo de observación, que es incluso más cercano que la historia local, al referirse al rescate de esos acontecimientos que están en las comunidades -como en el pueblo San José de Gracia- donde se está perdiendo la cultura y sus enseñanzas: la ordeña de las vacas, el procesamiento de la leche en quesos y requesón, las personalidades del pueblo y su contribución al desarrollo comunal, el centro ceremonial o iglesia, etc. Por microhistoria debemos entender - desde la postura de González- todo aquello que alcancemos a ver desde la torre de la iglesia, lo que se encuentra en el camino a casa y al trabajo; esto es necesario describirlo y darlo a conocer. Es entonces esta mirada desde el suelo, desde abajo, de la gene común, desde lo microscópico, desde el terruño en el que se nace o desde la matria, como lo expresa en algunas de sus obras don Luis González. Las discusiones fueron en realidad muy vastas y profundas, llegando en ocasiones a considerar que los trabajos que estaban realizando eran microhistorias: "La microhistoria de los murales de Chihuahua", "La microhistoria de los Colegios de Bachilleres de Chihuahua", "La microhistoria de los movimientos sociales en la Escuela Preparatoria de la Universidad de Chihuahua”, entre muchos otros.

Se abordó la vertiente de la microhistoria a la italiana; fue el doctor Carlos Rojas quien la definió en estos términos, según López (2005):

...es importante aclarar que el objetivo de esta microhistoria italiana no es el de estudiar las cosas pequeñas, ni las pequeñas anécdotas, ni tampoco los pequeños procesos. Ellos lo han dicho miles de veces: no estudiamos pueblos sino "en" los pueblos, pero también estudiamos por ejemplo obras de arte, o biografías de ciertos individuos, o a un sector de la clase obrera en Turín en un periodo de cincuenta años. Así que es un error total tratar de identificar a la microhistoria italiana con la simple historia local [p. 195].

La intención era diferenciar la microhistoria italiana y la mexicana. Para la primera se gestó una gran cantidad de ideas inspiradas en el padre de esta corriente, Carlo Ginzburg, quien no se dio cuenta de que su propuesta sería un parteaguas en la historiografía. Lo mismo ocurrió con don Luis González cuando acuñó el término, pues lo hizo con el fin de diferenciar la historia de su pueblo, que se perdía en esas historias nacionales o generales.

A la microhistoria de vertiente italiana le interesa el universo micro como el espacio para probar las hipótesis o supuestos previamente elaborados, es como el laboratorio para experimentar y confirmar. No está interesada en las cosas pequeñas y se aboca a los acontecimientos de más de 50 años de ocurrencia, estudia “en" los pueblos, y aquí es donde se encuentra otra diferencia con la vertiente mexicana, que estudia los pueblos y los describe, como ocurre con San José de Gracia. 
Para confirmar los enunciados de Ginzburg recurrimos a un texto que ya está muy trabajado entre los historiadores: "El queso y los gusanos: el cosmos de un molinero del siglo XVI" (Barbosa, 2015), en el cual se aprecia el juicio al que fue sometido Menocchio por la Santa Inquisición y posteriormente llevado a la hoguera al no obtener el perdón, por haberse obstinado en sus creencias. En esta obra se intenta conocer la cultura de la época a través de Menocchio el molinero, de dónde obtuvo esas ideas religiosas, cuáles eran las obras que circulaban y cómo habían forjado el pensamiento del molinero; además de conocer cómo actuaban los tribunales de la Santa Inquisición en defensa y quebranto contra la fe Católica; porque el universo imaginado por Menocchio no era resultado de ninguna persona común y corriente, y es allí donde también se prueba que no todos eran analfabetas como se había considerado, ya que nuestro personaje sabía leer y escribir, lo que estaba reservado para las clases sociales altas. El universo de Menocchio consideraba que al inicio era todo un caos: el agua, el aire, la tierra y el fuego juntos y revueltos se convirtieron en una gran masa, así como el queso se hace con la leche y al descomponerse nacen los gusanos. Estos se convirtieron en ángeles y uno de ellos -el más poderoso- se convirtió en dios, y de allí partió todo lo que se tiene en este mundo.

Otro ejemplo - con el fin de probar esos supuestos generalizadores- se encontró en el artículo "Brujería y piedad popular. Notas sobre un proceso de 1519 en Módena" (Saidón, 2018) que se ocupa de la existencia o no de la brujería. Realiza la narrativa de Chiara Signorini, una mujer campesina acusada de brujería, microhistoria surgida de los archivos de la Santa -o más bien no tan santa- Inquisición. Con estos ejemplos se cierra la visión de lo que aborda la microhistoria a la italiana y que también se ha conocido como historia cultural, precisamente porque a través de Menocchio o de Chiara se hace la narrativa y verificación de la cultura que se tenía en la época que se estudia; sin embargo, los debates ante esta visión de la historia cultural derivaron en Peter Burke (por motivos de espacio, no se profundizará aquí en este autor).

En relación a los métodos de la microhistoria, se encontró que don Luis González -a través de sus obras Pueblo en vilo: microhistoria de San José de Gracia, Invitación a la microhistoria y La nueva invitación a la microhistoria- rescata las historias del terruño, las personas y los recuerdos, todo ello a través de planteamientos emotivos, humanos, y es aquí donde surge la necesidad de recuperar la parte existencialista. Se consideró que el método es sencillo: búsqueda en archivos parroquiales y municipales, recuperación de oralidades y -sin perder lo humano- proceder a la narrativa. Se trata de una historia de la gente sencilla, simple, común; sin descuido de sus sentimientos, voliciones y deseos.

En relación a lo estudiado del paradigma de la microhistoria italiana, "plantea que la microhistoria realiza un proceso constructivo de la investigación para acceder al conocimiento científico. Sus trabajos están basados en un constructivismo consciente, en el cual no caben premisas dadas de antemano, ni se aceptan ciertas evidencias 
epistemológicas tradicionales" (Ronen, p. 168). El autor también menciona que es el "paradigma Indiciario" el que permite, a la manera de Charles Holmes, buscar las pistas necesarias para probar o disprobar el proyecto de investigación al que se están dirigiendo, y es el sujeto ese detective que recopila pruebas en el universo micro, en ese laboratorio de análisis de la ocurrencia del acontecimiento, como fue el caso relacionado con la brujería a nivel general y probado a nivel particular con el encuentro de Chiara Signorini en Módena, Italia, en el siglo XVI, acusada de brujería y de genuflexión ante el demonio.

Las ideas de los participantes en el seminario en relación a la metodología de la microhistoria llegaron a obtener el consenso, al considerar que para el rescate de los acontecimientos a nivel micro, ya sea desde la visión italiana o mexicana, es necesario buscar las pistas que alimentan los objetivos de la investigación para llegar a las fuentes; triangular información para describir, comprender y estar en posibilidades de hacer las narraciones correspondientes. No debe dejarse de lado que un indicio nos lleva a otros más ocultos y que no se debe perder de vista que el investigador (sujeto) es quien construye y reconstruye los acontecimientos para probar o disprobar en el nivel micro, el cual sirve de laboratorio experimental. Una característica más, es que no se debe perder en las búsquedas la esencia de lo humano.

Se deja aquí la descripción de lo que fue el Seminario de Microhistoria, que por supuesto fue mucho más allá de solo diferenciar lo concerniente a la microhistoria en las vertientes mexicana e italiana.

\section{CONCLUSIONES}

El Seminario de Microhistoria fue el espacio académico para la discusión propositiva de ideas, así como para tener elementos que blinden la investigación que están abordando los estudiantes del programa de doctorado en Educación, Artes y Humanidades.

Las lecturas seleccionadas para su análisis fueron el pretexto para derivar en otras reflexiones que tomaban rumbo con las discusiones.

En la conducción del seminario se asumió el rol del liderazgo compartido, en el cual cada participante tuvo la oportunidad de dirigir la sesión de trabajo.

Lo narrado en el presente artículo solo fue una temática encaminada a diferenciar teóricamente las vertientes mexicana e italiana, lo que se realizó con éxito debido a los comentarios al final de las actividades.

Se considera que los seminarios, en el estricto apego al semen, germen o semilla de las discusiones con sentido, contribuyen a la generación de una discusión de altura, incluyente, así como a la internalización y construcción de otras ideas innovadoras, como ocurrió con el seminario que aquí se describe.

Finalmente se considera que el seminario dio respuesta a los objetivos planteados y superados ante los hallazgos en el desarrollo del mismo, además de generar una actitud de compromiso y entrega en la edificación de los conocimientos relacionados 
con la apropiación de las herramientas teórico-metodológicas que aporta el paradigma de la microhistoria.

\section{AgradeCIMIENTOS}

Se agradece a la Universidad Autónoma de Chihuahua y en especial a las autoridades de la Facultad de Filosofía y Letras por todos los apoyos que brindaron para elaborar y presentar el presente artículo.

\section{REFERENCIAS}

Barbosa, W. (2015). De Menocchio a la imaginaria política. El Tranvía. Revista Digital de Análisis Politico y Cultural. Recuperado de: http:/ / revistaeltranvia.com.ar/de-menocchioa-la-imagineria-politica/.

Jenkins, K. (2006). ¿Por qué la historia? Ética y posmodernidad. México: Fondo de Cultura Económica.

Lafuente, María (1998). Introducción al pensamiento de Paul Ricoeur. Thémata. Revista de Filosofia, (19), pp. 219-223.

López, C. (2005). Mesa redonda: microhistoria mexicana, microhistoria italiana e historia regional. Relaciones, 26(101), 193-224.

Ronen, M. (2013). La microhistoria como referente teórico-metodológico. Un recorrido por sus vertientes y debates conceptuales. Historia actual online, (30), 167-173.

Saidón, G. (2018). Carlo Ginz̧burg: secretos de un bistoriador que busca no aburrir a los lectores. Infobae. Recuperado de: https://www.infobae.com/america/cultura-america/2018/10/16/ carlo-ginzburg-la-historia-y-la-literatura-se-retroalimentan/.

White, H. (1992). Metahistoria. La imaginación histórica en la Europa del siglo XIX [trad. S. Mastrangello]. México: FCE.

Cómo citar este artículo:

Pérez Piñón, F. A., Hernández Orozco, G., y Liddiard Cárdenas, S. (2020). Historia de la educación en Chihuahua: el Seminario de Microhistoria. RECIE. Revista Electrónica Científica de Investigación Educativa, 5(1), pp. 277-285. doi: doi.org/10.33010/recie.v5i1.949. 